\title{
Electroacupuncture therapy in inflammation regulation: current perspectives
} This article was published in the following Dove Press journal:
Journal of Inflammation Research

\section{Ji-Yeun Park \\ Uk Namgung}

Department of Oriental Medicine, Daejeon University, Daejeon, South Korea
Corresponding author: Uk Namgung Department of Oriental Medicine, Daejeon University, 62 Daehak-ro, Yongun-dong, Dong-gu, Daejeon 34520, South Korea

Tel +82 422802614

Email unamgung@dju.kr

\begin{abstract}
Although acupuncture therapy is increasingly used to treat diverse symptoms and disorders in humans, its underlying mechanism is not known well. Only recently have experimental studies begun to provide insights into how acupuncture stimulation generates and relates to pathophysiological responsiveness. Acupuncture intervention is frequently used to control pathologic symptoms in several visceral organs, and a growing number of studies using experimental animal models suggest that acupuncture stimulation may be involved in inducing anti-inflammatory responses. The vagus nerve, a principal parasympathetic nerve connecting neurons in the central nervous system to cardiovascular systems and a majority of visceral organs, is known to modulate neuroimmune communication and anti-inflammatory responses in target organs. Here, we review a broad range of experimental studies demonstrating anti-inflammatory effects of electroacupuncture in pathologic animal models of cardiovascular and visceral organs and also ischemic brains. Then, we provide recent progress on the role of autonomic nerve activity in anti-inflammation mediated by electroacupuncture. We also discuss a perspective on the role of sensory signals generated by acupuncture stimulation, which may induce a neural code unique to acupuncture in the central nervous system.
\end{abstract}

Keywords: electroacupuncture, anti-inflammation, vagus nerve, animal model, acupuncture mechanism

\section{Introduction}

Acupuncture has been used as a traditional medical treatment in East Asia for over 2,000 years, ${ }^{1}$ and is becoming a popular therapy worldwide for treating various diseases. ${ }^{2,3}$ Acupuncture is a medical intervention in which fine needles are applied to specific parts of the body, called acupuncture points (or acupoints) and penetrated through the muscular or other subcutaneous layers. According to traditional medical theory, acupuncture stimulation facilitates the flow of qi, a life force that is supposedly circulating through the channels called meridians. ${ }^{4,5}$ Acupoints are presumed to be pathophysiologically associated with and possibly reflect the status of visceral organs and systemic conditions, and thus the stimulation of specific acupoints may evoke the responsiveness that controls the unbalanced internal milieu and improves body symptoms. Acupuncture stimulation is given right on the acupoint or a nearby affected area ("ashi point") for the treatment of local symptoms, such as knee pain or muscle rigidity, whereas distal acupuncture stimulation is applied to treat diseases in the internal organs and systemic abnormalities. 
There are two main types of acupuncture stimulation: manual acupuncture (MA) and electroacupuncture (EA). In MA, an acupuncturist penetrates the skin with a metallic needle and manipulates it by rotating in one or both directions or lifting and thrusting. ${ }^{6}$ It is known that during acupuncture practice, acupuncturists experience a special touch sensation perceived as heaviness, tenseness, or terseness, and patients perceive feelings of numbness, heaviness, soreness, and distention around the site of needle stimulation. These are called deqi sensations. Clinical data further indicate that patients frequently feel deqi sensations spreading to other parts of the body, ${ }^{7-9}$ which is considered a useful criterion to evaluate the therapeutic efficacy of acupuncture. ${ }^{710-12}$ In EA, a small electric current is applied to pairs of acupuncture needles, and studies have indicated that the therapeutic efficacy of EA can be modulated by varying the frequency, intensity, and duration of electrical stimulation. ${ }^{6} 13$ For instance, EA at low and high frequencies of electrical stimulation can activate different types of opioid receptors and different analgesic effects. ${ }^{14,15}$ To maximize therapeutic effects, acupuncture is usually practiced first by applying MA to evoke deqi sensation and followed by electrical stimulation for 15-20 minutes. ${ }^{7}$

A growing number of recent reports have indicated that acupuncture may be effective in treating many types of diseases by regulating inflammatory responses. In this review, we highlight important findings that demonstrate how acupuncture stimulation, particularly EA, can improve inflammatory responses in pathological animal models. First, we discuss recent advances in understanding of neuroimmune communication, and then address how it has contributed to establishing experimental approaches to investigate a mechanistic basis of EA. In the last part of the review, we briefly discuss a perspective on the role of a neural code that may transmit sensory information unique to acupuncture in regulating inflammation.

\section{Neuroimmune communication}

When an organism is exposed to external pathogens, hostdefense response begins with innate immunity, which is critical to induce inflammation as a defense mechanism against pathogenic infections. Pathogen-associated molecular patterns trigger inflammatory reactions in the host via interaction with membranous or cytoplasmic molecules, such as Toll-like receptors (TLRs), NOD-like receptors (NLRs), Retinoic acid-inducible gene 1-like receptors (RLRs), and C-type lectin receptors (CLRs), collectively termed "pattern-recognition receptors". ${ }^{16}$ Activation of these receptors in target cells induces downstream-signaling pathways, including activation of the MAPK pathway and $\mathrm{NF} \kappa \mathrm{B}$ transcription factor, and induces the expression of inflammatory cytokines, including TNF $\alpha$ and several types of interleukins and chemokines. Chemokines recruit leukocytes into the inflammation area, and interleukins and IFN $\gamma$ activate lymphocytes and macrophages.

\section{Cholinergic anti-inflammatory reflex}

In the early 2000s, Borovikova et al reported on their seminal work on the regulation of inflammatory responses by vagus nerve activity. They found that the electrical stimulation of the vagus nerve in vivo decreased the production of TNF $\alpha$ in the spleen of lipopolysaccharide (LPS)-injected animals, and treatment with acetylcholine of cultured macrophages attenuated levels of inflammatory cytokines as well. ${ }^{17}$ They further demonstrated that the suppression of TNF $\alpha$ production by acetylcholine treatment was mediated by the activation of $\alpha_{7}$-nicotinic acetylcholine receptors, which subsequently inhibited NFKB activation while stimulating the STAT3 pathway. ${ }^{18-20}$ The vagus nerve is known to account for $70 \%$ of the parasympathetic, visceral regulation of internal organs, thus acting as a functional bridge connecting the brain to internal organs. Inflammatory cytokines produced from peripheral organs can activate an afferent part of the vagus nerve, stimulate vagal efferent nerves through the synaptic transmission from the solitary nucleus to the dorsal vagal nucleus in the brain stem, and downregulate the production of inflammatory cytokines, thereby completing the cholinergic anti-inflammatory reflex. ${ }^{21}$ Rosas-Ballina et al reported that the vagal efferent nerves are connected to adrenergic postganglionic neurons in the celiac ganglion, which primarily receive splanchnic preganglionic sympathetic inputs from the spinal cord, ${ }^{22}$ and further showed that norepinephrine released from celiac ganglion neurons stimulated the secretion of acetylcholine from choline acetyltransferase-expressing $\mathrm{T}$ cells in the spleen that binds to nicotinic acetylcholine receptors in macrophages. ${ }^{23}$ Similar experimental strategies investigating the effects of vagus nerve activity on the pathologic regulation of several conditions, such as arthritis, obesity, and head trauma, have been considered for clinical application, and the anti-inflammatory reflex is becoming accepted as a concept explaining neuroimmunogenic control of diseases. ${ }^{24}$

Neuroimmunogenic regulation of inflammation further provides insights into studies on how brain activity modulates pathological responsiveness in major internal organs. For 
instance, mental activities, such as stress, biofeedback therapy, and meditation, have been reported to be positively related to increased vagus nerve activity. ${ }^{23}$ It has also been reported that impaired vagal activity increased the vulnerability of inflammatory bowel disease in an animal model of depression. ${ }^{25}$

It should however be noted that the connectivity between the terminals of autonomic fiber and the target immune cells has not been clearly demonstrated in several organs. While increasing numbers of publications have reported on the role of vagal activity in hepatic hypertension and inflammation based on the concept of cholinergic anti-inflammatory reflex, ${ }^{17,26,27}$ hepatic target cells of vagal efferent fibers have not been identified. ${ }^{28,29}$ Similarly, autonomic connections to the spleen are unclear and controversial. In this regard, it is worthwhile to note one recent report showing that the electrophysiological and histological identification of serotoninsecreting enterochromaffin cells in the intestine that modulate synaptically connected afferent nerve fibers can fulfill the minimal requirement of brain-gut communication. ${ }^{30}$

\section{Functional intervention of sympathetic activity}

While the vagus nerve has been a primary target mediating neuroimmune reaction in many studies, a potential role of sympathetic nerve activity has also been proposed. Martelli et al claimed that neurons from celiac ganglia that are primarily innervated by sympathetic splanchnic nerves were responsible for anti-inflammatory reflexes. ${ }^{31,32}$ Adrenergic inputs activated hepatic invariant natural killer T (iNKT) cells and elevated the production of anti-inflammatory cytokines from T-helper 2 cells, rendering the organ more susceptible to infection via immunosuppression. ${ }^{33}$ Possible connections of sympathetic nerve activity to immune cells in target organs, such as liver and spleen, for the regulation of inflammatory reactions are depicted in Figure 1.

\section{Experimental studies on EA using pathologic animal models}

As a modified version of traditional MA, EA is manipulated at the same acupuncture points as MA, but electric current is additionally applied. Needle rotation, which is performed routinely during MA, can result in mechanical deformation of dermal tissue and may activate special types of mechanosensory receptors (eg, Ruffini corpuscles). Previous studies have suggested that acupuncture-specific responses, such as the production of mechanical torque and induction of specific types of integrin proteins, are related to needle rotation. ${ }^{34,35}$ In contrast, electric current, which is applied in EA, would spread to a nearby area and affect the peripheral nerve pulses (ie, action potential) more intensely, which may act as a possible reason to explain a certain level of therapeutic effects caused by sham EA, as reported in human subjects, ${ }^{36}$ yet in other studies, sham EA stimulation (EAS) was less effective than a combined manipulation of MA and EA. ${ }^{37,38}$

It has been reported that MA can attenuate inflammatory responses by regulating the production of IL10 in macrophages, hypothalamic expression of IL1 $\beta$ and IL6 mRNA, and serum TNF $\alpha$ production in LPS-injected animals. ${ }^{39-41}$ However, edema response by capsaicin injection is reduced by EA, but not by MA. ${ }^{42}$ Since the vast majority of studies on the regulation of inflammation have been conducted using EA, here we focus primarily on EA studies unless otherwise indicated.

\section{Anti-inflammatory effects of EA in animal models}

A study using animals given radiant heat on the tail identified gene groups (neurotransmitter-related genes vs proinflammatory cytokine-related genes) showing differential regulation of expression in the spinal dorsal horn after

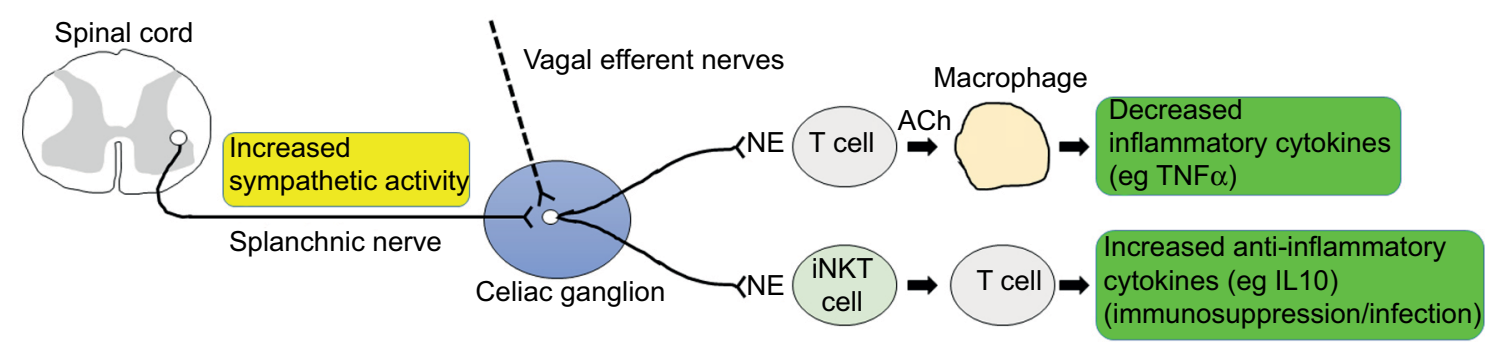

Figure I Sympathetic connection to immune cells in target organs.

Notes: Splanchnic nerve activity transmitted to the celiac ganglion may increase the release of norepinephrine (NE) of adrenergic postganglionic neurons. This in turn would activate immune-cell responses in target organs, such as macrophages in the spleen and $\mathrm{T}_{\mathrm{H}} 2$ cells in the liver, and these cells regulate the production of pro- and antiinflammatory cytokines. Vagal input, which has not been clearly identified, may have similar effects on the regulation of inflammation as sympathetic activity. Abbreviations: $\mathrm{T}_{\mathrm{H}}$, $\mathrm{T}$ helper; ACh, acetylcholine; iNKT, invariant natural killer $\mathrm{T}$. 
$\mathrm{EA}^{43}$ suggesting that EA may be involved in the regulation of inflammation at gene-expression level. In order to investigate the effects of EA on the regulation of inflammation, injury models of complete Freund's adjuvant (CFA)induced inflammation, collagen-induced inflammation, cerebral ischemia, reperfusion injury, and others have been used. The CFA-inflammatory model is frequently used to investigate inflammation-related pain and is thus useful to investigate pain regulation by EA. EA manipulation efficiently suppresses glial cell-marker proteins and TRPV1 and attenuates pain responses. ${ }^{44,45}$ EA also suppresses edema in CFA-inflammation animals by activating corticotropinreleasing hormone-producing neurons in the hypothalamus and increasing levels of adrenocorticotropic hormone. ${ }^{46}$ In an animal model of collagen-induced arthritis, EA attenuated inflammatory pain via the mediation of cholinergic and serotonergic receptors and also attenuated the production of inflammatory cytokines, such as IL1 $\beta,-6$, and -8, TNF $\alpha$, and NFאB in synovial tissue. ${ }^{47,48}$ Moreover, EA was effective in regulating the levels of TNF $\alpha$, IL1 $\beta$, IL6, and myeloperoxidase in animal models of ulcerative colitis and zymosan-induced acute arthritis, ${ }^{13,49,50}$ increased superoxide dismutase, while reducing death-related proteins, such as caspase 3, and phosphorylation of p38 and JNK in animals with cardiopulmonary bypass-induced lung injury, ${ }^{6}$ and suppressed NF $\kappa$ B in a rat-tissue chamber model of inflammation. ${ }^{51}$ In an acute alcoholic liver-injury model, EA improved hepatic circulation and adjusted ALT and AST levels. ${ }^{52}$ Finally, systemic inflammatory response and survival rate are significantly improved by EA in animals injected with a lethal dose of LPS. ${ }^{53}$ Major studies on the regulation of inflammation by EA are summarized in Table 1 .

Table I Summary of electroacupuncture (EA) studies on the regulation of inflammation in experimental animals

\begin{tabular}{|c|c|c|c|}
\hline Animal model & Acupoints & Major EA effects & Reference(s) \\
\hline $\begin{array}{l}\text { Intestinal ischemia- } \\
\text { reperfusion injury }\end{array}$ & $\begin{array}{l}\text { ST36, ST36 } \\
\text { plus stem-cell } \\
\text { transplantation }\end{array}$ & $\begin{array}{l}\text { Increased crypt-cell-proliferation index and mucosal mRNA expression of } \\
\text { SDFI, CXCR4, EGF, and EGFR } \\
\text { Decreased mucosal NFKB, p65 and serum inflammation factor (TNF } \alpha \text {, IL6) }\end{array}$ & 97 \\
\hline $\begin{array}{l}\text { Spinal cord ischemia- } \\
\text { reperfusion injury }\end{array}$ & GV6, GV9, EX-B2 & $\begin{array}{l}\text { Decreased TNF } \alpha \text {, ILI } \beta \text {, and MMP9 } \\
\text { Neuroprotective effects of EA suppressed by autophagic inhibitor } \\
\text { (3-methyladenine) }\end{array}$ & 98 \\
\hline \multirow[t]{2}{*}{ TNBS-induced colitis } & ST36, ST36 plus VNS & $\begin{array}{l}\text { Decreased TNF } \alpha \text {, ILI } \beta \text {, IL6, and MPO } \\
\text { Increased vagal activity and decreased sympathetic activity }\end{array}$ & 13 \\
\hline & PC3 and PC6 & Decreased ILI $\beta$ and NFKB & 99 \\
\hline CPB-induced lung injury & PC6 and LI4 & $\begin{array}{l}\text { Decreased } \mathrm{pP} 38, \mathrm{pJNK} \text {, and caspase } 3 \\
\text { Increased SOD activity }\end{array}$ & 6 \\
\hline $\begin{array}{l}\text { Thermal injury-induced } \\
\text { remote acute lung injury }\end{array}$ & ST36 & Decreased ILI $\beta$, IL6, and HMGBI & 100 \\
\hline \multirow[t]{5}{*}{ CFA-induced inflammation } & ST36-ST37 & $\begin{array}{l}\text { Decreased TRPVI, pERK, pp } 38 \text {, pJNK, pAkt, pCREB, NavI.7, and Navl.8 in } \\
\text { DRG and Schwann cells }\end{array}$ & 44,45 \\
\hline & ST36 & $\begin{array}{l}\text { Decreased GFAP, IBAI, SI00ß, RAGE, and TRPVI, in the DRG and spinal } \\
\text { cord dorsal horn } \\
\text { Blocking opioid and adenosine } \mathrm{A}_{1} \text { receptors reversed the effects of EA }\end{array}$ & 45 \\
\hline & ST36 and GB34 & Increased apelin, APJ protein, and mRNA expression in the spinal cord & 101 \\
\hline & ST36 and BL60 & $\begin{array}{l}\text { Decreased mRNA and protein levels of TLR4, MYD88, and NFKB in ankle- } \\
\text { bone tissue }\end{array}$ & 102 \\
\hline & GB30 & $\begin{array}{l}\text { Increased plasma ACTH levels } \\
\text { Increased phosphorylation of NRI in CRH-containing neurons in the PVN }\end{array}$ & 46 \\
\hline $\begin{array}{l}\text { Electrical stimulation- } \\
\text { induced migraine }\end{array}$ & GB20 and TE5 & $\begin{array}{l}\text { Decreased serum CGRP and PGE2 } \\
\text { Decreased ILI } \beta \text { and COX2 expression in the trigeminal ganglion } \\
\text { Decreased plasma protein extravasation } \\
\text { CBI-receptor antagonism reversed the effects of EA }\end{array}$ & 103 \\
\hline Septic brain injury & GV20, ST36 & $\begin{array}{l}\text { Decreased TNF } \alpha \text {, IL6, and MDA } \\
\text { Increased SOD and catalase activities in the serum and hippocampus } \\
\text { Decreased TLR4, NFKB and IBAI expression }\end{array}$ & 104 \\
\hline $\begin{array}{l}\text { MPTP-induced Parkinson's } \\
\text { disease }\end{array}$ & ST36 and SP6 & $\begin{array}{l}\text { Increased human placental alkaline phosphatase } \\
\text { Decreased microglia activation in the striatum and midbrain }\end{array}$ & 87 \\
\hline
\end{tabular}


Table I (Continued)

\begin{tabular}{|c|c|c|c|}
\hline Animal model & Acupoints & Major EA effects & Reference(s) \\
\hline \multirow[t]{6}{*}{ Obesity } & $\mathrm{CVI} 2$ and $\mathrm{CV} 4$ & Decreased serum TNF $\alpha$ & 105 \\
\hline & ST36 & Decreased adipose tissue inflammation & 106 \\
\hline & & Decreased HIF $\alpha$, hypoxia-related genes (VEGFA, SLC2AL, and GPXI), and & \\
\hline & & inflammation-related genes (TNFA, IL6, and MCPI) & \\
\hline & & Decreased macrophage recruitment and infiltration & \\
\hline & & Decreased NFKB and increased $I \kappa B \alpha$ & \\
\hline Collagen-induced & ST36, GB39, BL23 & Decreased levels of NFKB (p65), TNF $\alpha$, ILI $\beta$, IL6, and IL8 & 48 \\
\hline \multirow[t]{3}{*}{ rheumatoid arthritis } & GB39, ST36 & Increased mRNA expression of VPACI & 107 \\
\hline & & $\begin{array}{l}\text { Elevated } \mathrm{CD} 4^{+} \mathrm{FOXP3^{+ }} \mathrm{T}_{\text {reg }} \text {-cell frequency and reduced } \mathrm{CD} 4^{+} \mathrm{ILI} / 7^{+} \mathrm{T}_{H} 17 \text {-cell } \\
\text { frequency }\end{array}$ & \\
\hline & ST36 & $\begin{array}{l}\text { Analgesic effect of EA was mediated by } m A C h R, 5 \mathrm{HT}_{1 \mathrm{~A}} \text {, and } 5 \mathrm{HT}_{3} \\
\text { receptors, but not by } 5 \mathrm{HT}_{2} \text { receptor }\end{array}$ & 47 \\
\hline Ligature-induced & LI4, LIII, ST36, ST44 & Decreased TRAP-positive multinucleated cells & 108 \\
\hline \multirow[t]{2}{*}{ periodontitis } & & Decreased expression of ILI $\beta$ and MMP8 mRNAs & \\
\hline & & Increased expression of IL6 mRNA & \\
\hline Cerebral ischemia- & GV20 and ST36 & Decreased ACTH and HSP70 & 2 \\
\hline \multirow[t]{13}{*}{ reperfusion injury } & GV20 and GVI4 & Increased rCBF and IL6 expression & 109 \\
\hline & & Decreased ILI $\beta$ and JAK2 & \\
\hline & GV20 and GVI4 & $\begin{array}{l}\text { Decreased mRNA level of ChAT, five subtypes of muscarinic receptors and } \\
\alpha_{7} n A C h R\end{array}$ & 92 \\
\hline & GV20 and GVI4 & Decreased Bax, TNF $\alpha$, IL6, and ILI $\beta$ & 110 \\
\hline & & Decreased excitotoxicity by NMDA & \\
\hline & & Increased antioxidant systems (Bcl2, Nrf2, GCSh, GCSI, Gsh) & \\
\hline & LIII and ST36 & Decreased microglia activation of IBAI and EDI in cortex & 78,111 \\
\hline & & Decreased serum TNF $\alpha$, ILI $\beta$, and IL6 & \\
\hline & & Decreased cortical p38 MAPK and MyD88 & \\
\hline & & Decreased cortical NFKB, TNF $\alpha$, and ILI $\beta$ & \\
\hline & GV20 and ST36 & $\begin{array}{l}\text { Inhibited neuronal apoptosis, microglial activation of IBAI, and oxidative } \\
\text { stress in the hippocampus }\end{array}$ & 79 \\
\hline & & Decreased hippocampal and serum IL6 and TNF $\alpha$ levels & \\
\hline & LIII and ST36 & Decreased TLR4/NFKB signaling and levels of TNF $\alpha$, ILI $\beta$, and IL6 & 112 \\
\hline
\end{tabular}

Abbreviations: HT, hydroxytryptamine; ACTH, adrenocorticotrophic hormone; APJ, apelin receptor; CFA, complete Freund's adjuvant; CGRP, calcitonin gene-related peptide; CPB, cardiopulmonary bypass; CRH, corticotrophin-releasing hormone; DRG, dorsal root ganglion; Gsh, glutathione; MDA, malondialdehyde; MPTP, I-methyl-4phenyl-I,2,3,6-tetrahydropyridine; NMDA, N-Methyl-d-aspartic acid; PGE2, prostaglandin $\mathrm{E}_{2}$; PVN, paraventricular nucleus; rCBF, regional cerebral blood flow; TG, trigeminal ganglion; TNBS, 2,4,6-trinitrobenzenesulfonic acid.

\section{EA-related signaling events in the nervous system}

While an increasing number of reports strongly indicate that EA can regulate inflammation and associated pathologic symptoms, underlying mechanisms remain largely elusive. Considering that acupuncture and meridian networks encompass the whole body, as does the neural network, it is not surprising that the nervous system has been a primary concern for mechanistic studies on acupuncture. Indeed, a growing number of studies have supported this notion. Cutaneous acupuncture at the zusanli acupoint has been reported to elevate levels of focal adenosine and its $A_{1}$ receptor for antinociceptive actions ${ }^{54}$ and upregulate the expression of purinergic receptors in the dorsal root ganglion, ${ }^{35,55}$ suggesting acupuncture-mediated induction of afferent signals. It has been further reported that acupuncture stimulation induced c-Fos expression in neurons in the dorsal vagal complex area, including both nucleus tractus solitarii (NTS) and dorsal motor nucleus, rostral ventromedial medulla, and raphe nucleus, implying that the ascending neuronal signals generated from the cutaneous acupoints relay synaptic inputs into the vagal neural circuits and possibly further up to the cerebral neural circuits. ${ }^{39,56-58}$

Evidence demonstrating the involvement of autonomic nerve activity in acupuncture action largely comes from physiological studies analyzing cardiovascular responses and gastrointestinal (GI) motility in experimental animals and humans. Physiological assessment of GI activity is useful to investigate acupuncture effects on autonomic function, because the regulation of GI disorders is one of the major realms of traditional acupuncture medicine ${ }^{59,60}$ One study reported that the manipulation of EA in dogs improved 
gastric emptying and increased vagal activity assessed by spectral analysis of heart-rate variability while suppressing sympathetic activity. ${ }^{61}$ Acupuncture effects on regulation of heart-rate variability were similarly demonstrated in a rodent model of inflammatory bowel disease and human subjects. ${ }^{13,62}$ Longhurst and Tjen-A-Looi reported that acupuncture stimulation regulated cardiovascular function (blood pressure), whereby neurotransmitter release and neuromodulation in the hypothalamus and several cardiovascular nuclei in the brain stem mediated acupuncture effects. ${ }^{63}$

In addition to nuclei in the vagal complex, EA was reported to induce neuronal activation in the cerebrum. Functional magnetic resonance-imaging studies using human subjects revealed that analgesic effects caused by low-frequency EA ( $2 \mathrm{~Hz}$ ) were positively correlated with activation in the contralateral primary motor area, supplementary motor area, and ipsilateral superior temporal gyrus, but negatively correlated in the bilateral hippocampus. ${ }^{64}$ However, analgesic effects induced by high-frequency EA $(100 \mathrm{~Hz})$ were positively correlated with activation in other areas, such as the contralateral inferior parietal lobule, ipsilateral anterior cingulate cortex, nucleus accumbens, and pons. In another functional magnetic resonance-imaging study, EA activated neurons in the cingulate cortex and modulated the activation pattern of limbic-system networks. ${ }^{65}$

\section{Acupuncture mechanism on anti- inflammation: potential role of vagus nerve activity}

Based on the preceding discussion, it is likely that EA generates neuronal signals at the acupuncture point, sends these to the spinal cord and brain, and may trigger autonomic regulation of inflammatory responses in target organs. How would EA-induced sympathetic and parasympathetic nerve activities modulate pathological responsiveness in internal organs? Here, we discuss the role of vagus nerve activity as a principal parasympathetic nerve and then sympathetic nerve activity.

As discussed in the previous section, vagus nerve stimulation (VNS) activates nicotinic acetylcholine receptors in target cells to attenuate inflammatory responses. Here, one intriguing issue is whether the vagus nerve activity induced by EAS is functionally comparable to the VNS. It should be noted that since the VNS acts on both afferent and efferent parts of the nerve, afferent signals transmitted to the brain could indirectly affect inflammatory responses. Likewise, EA signals, if any, would be transmitted directly to the vagal efferent nerves or indirectly by way of brain pathways, and thus the vagal activity caused by EAS and VNS may share some common neural code in acting on a target organ (Figure 2). Previous studies have shown that EA given at several different acupoints activates neurons in the NTS, a brain-stem location receiving somatic nerve activity and afferent vagal inputs, as demonstrated by c-Fos immunostaining and electrophysiological recordings. ${ }^{57,66,67}$ EAS-induced neuronal activity in the NTS would send out signals to vagal efferent nerves or ascending neural paths to upper-brain neurons, and combined efferent activity may mediate anti-inflammatory effects (Figure 2). In this respect, one previous study demonstrating the involvement of brain muscarinic receptor-mediated networks in anti-inflammatory regulation of EA in LPS-induced endotoxemia ${ }^{53}$ supports the notion that EAS-induced efferent vagal activity may carry the descending brain activity as acupuncture-specific neuronal signals.

Torres-Rosas et al demonstrated that sciatic nerve activation by EA at the zusanli acupoint inhibited the production of major inflammatory cytokines in an animal model of polymicrobial peritonitis, and further showed that vagal activity transmitted to the adrenal gland increased dopamine

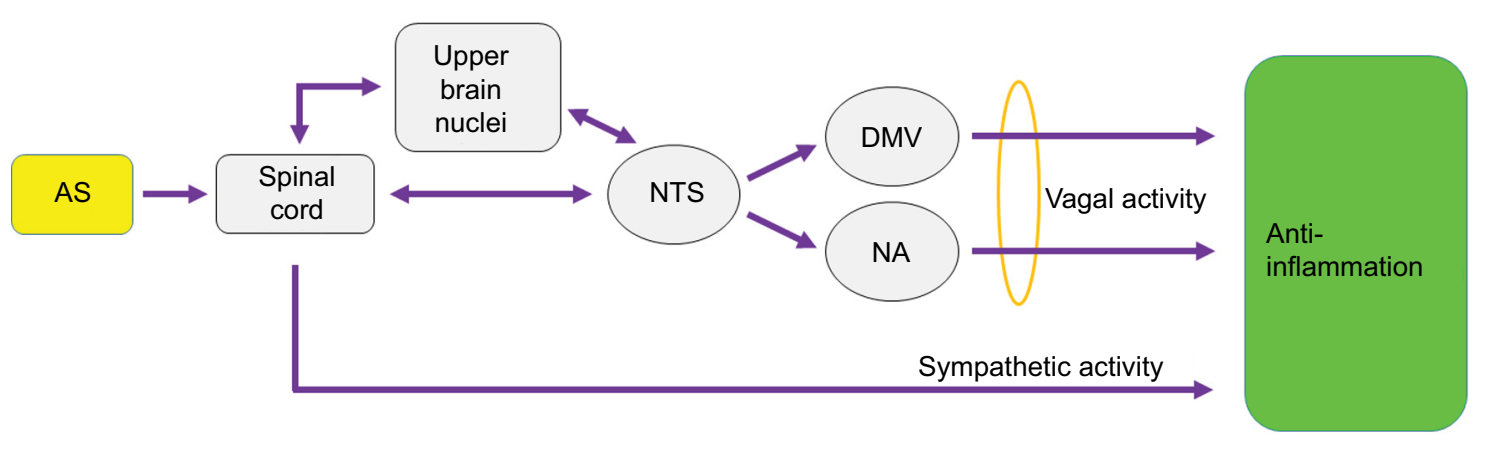

Figure 2 Possible neural pathways that transmit cutaneous ASs ultimately to internal visceral organs and induce anti-inflammatory responses in target organs. Abbreviations: AS, acupuncture signal; NTS, nucleus tractus solitarius; DMV, dorsal motor nucleus of vagus nerve; NA, nucleus ambiguus. 
production and downregulated cytokine production. ${ }^{68}$ However, in another study using LPS-induced systemic inflammation animals, EA at the zusanli acupoint activated the vagal pathway connected to the spleen and attenuated the production of TNF $\alpha$ in the spleen. ${ }^{39}$ In an animal-sepsis model with a lethal dose of LPS, vagotomy abrogated the effects of EAS (at the hegu acupoint) on anti-inflammation and animal survival, ${ }^{53}$ suggesting the activation of vagal efferent by EAS. However, EAS (at the zusanli acupoint) activated NTS neurons and improved the pathologic parameters of postoperative ileus, but did not regulate the production of inflammatory cytokines. ${ }^{66} \mathrm{In}$ ischemia-reperfusion animals, vagotomy or administration of nicotinic receptor antagonist reversed EA inhibition on the release of HMGB1 and myocardial protection. ${ }^{69}$ Finally, in a TNBS-induced colitis model, when given together with VNS, EAS effectively decreased the production of inflammatory cytokines and pathogenesis in the colon, but EAS alone did not increase vagal activity above VNS, ${ }^{13}$ implying some augmentation effects of EAS on VNS-mediated anti-inflammation. It seems evident that vagal activity is a principal modulating factor for regulation of inflammation by EA; however, further studies are essential to verify vagal modulation of EA in different disease models using various EAS manipulations.

\section{Anti-inflammatory regulation of EA via sympathetic nerve pathway}

Possible involvement of sympathetic nerve activity in neuroimmune regulation has been implicated by adrenergic neuronal activity inducing iNKT cells, whose inhibition attenuated immunosuppression and bacterial infection (Figure 1). ${ }^{33}$ Can a concept of anti-inflammatory regulation via the suppression of sympathetic activity be applied to acupuncture-mediated anti-inflammation? Previous reports have suggested that in a physiological state, EA may either increase or decrease sympathetic nerve activity in several organ systems. For instance, EAS improves rectal motility by inhibiting sympathetic nerve activity, but suppresses gastric motility by increasing sympathetic activity. ${ }^{70,71} \mathrm{EA}$ increases cellular uptake of glucose by increasing sympathetic nerve activity. ${ }^{72}$ It has further been reported that EA was involved in cardiovascular baroreceptor reflex by modulating hypothalamic inputs to rostroventrolateral medulla, an area relaying signals to preganglionic sympathetic neurons in the spinal cord. ${ }^{73}$ In relation to inflammatory responses under a pathological state, EA attenuates inflammatory reaction by activating postganglionic sympathetic nerve activity in carrageenan- and zymosan-induced animal models. ${ }^{74,75}$
However, in LPS-induced endotoxemia animals, EA inhibits peripheral sympathetic activity and increases vagal activity to regulate systemic inflammatory responses. ${ }^{53}$ This suggests that (possibly reflecting differential experimental systems) either increased or decreased sympathetic nerve activities are involved in mediating anti-inflammatory effects of EA.

Martelli et al claimed that instead of vagus nerve activity, sympathetic activity, which is evoked by electrical stimulation of splanchnic nerve connected to celiac ganglia, might be responsible for regulating anti-inflammatory response in the spleen. ${ }^{31,32}$ Therefore, it is tempting to explore the possibility that anti-inflammation by EAS is modulated by splanchnic nerve activity transmitted to celiac ganglia. Immune cells, such as iNKT cells, and a subpopulation of $\mathrm{CD}^{+} \mathrm{T}$ cells are known to receive adrenergic inputs from sympathetic nerve terminals and mediate immunosuppression and inflammation (Figure 1). ${ }^{23,33}$ Therefore, one experimental approach to explore the mechanistic basis of the regulation of sympathetic nerve activity by EA would be to investigate whether EAS activates preganglionic neurons in the spinal cord and subsequent celiac ganglia neurons, and analyze the activation of immune cells in pathologic target organs, such as spleen and liver, in association with sympathetic nerve connectivity.

\section{Regulation of ischemic brain injury by EA}

EA has been widely used for the treatment of stroke and cerebral ischemia. ${ }^{76}$ To understand the biological basis for its efficacy, rodent models of cerebral ischemia-reperfusion and middle cerebral artery occlusion are used widely. Histological and behavioral examinations demonstrated that EA manipulation protected neural tissue from injury and improved motor impairment and cognitive function. ${ }^{77-81}$ Studies at cellular and molecular levels showed that EAS regulated the expression of apoptosis-related genes, such as $B C L 2$ and $B A X,{ }^{77,82}$ inhibited the production of HMGB1 production, ${ }^{83,84}$ known to induce inflammation by activating TLR 4 and RAGE, ${ }^{85}$ and downregulated mRNA and protein levels of MMP2, Aqp4, and Aqp9 ${ }^{86,87}$ EAS also activates several signaling molecules and related pathways, including stimulation of STAT ${ }^{88}$ and PI3K, ${ }^{89}$ and upregulates levels of glutamate receptor GluR2 for neuroprotection. ${ }^{90}$ Finally, EA affects the activation of microglial cells and astrocytes and the production of astroglial lactate transporter (MCT1), implying that EA may exert its protective function by acting on glial cells and neurons in the central nervous system. ${ }^{78,80,81,91}$

Despite numerous reports on pathologic responses by EA, mechanistic studies underlying acupuncture efficacy on 
cerebral ischemia have been limited. Chavez et al described ${ }^{76}$ on a mechanistic basis a list of possible beneficial effectors of acupuncture (both EA and MA) in brain areas after ischemic stroke. In an experimental stroke model, EAS has been shown to attenuate cerebral ischemia by activating $\alpha_{7}$-nicotinic acetylcholine receptors in penumbra and also vagal motor neurons. ${ }^{92}$ However, it is unclear how EA-modulated vagal activity is linked to cholinergic nerve activity in ischemic cerebral tissue and leads to pathological responsiveness in target tissue. Further studies demonstrating the protective effects of EAS on brain and spinal tissue from ischemic damage are critical to gain insights into the mechanistic basis of anti-inflammatory mechanisms of EA.

\section{Perspectives: neural coding of acupuncture signals}

All sensory information in humans and animals is received by specific receptors, and the nerve signals generated are transmitted to and perceived by the brain. Modality, location, intensity, and duration are basic features of sensory stimulation at the periphery, and act as basic elements to transduce unique signals to sensory neurons in the brain. ${ }^{93}$ Here, the initial stimulation requires the process of neural coding so that the sensory signals can be interpreted through brain circuitry. ${ }^{94}$

Would cutaneous acupuncture stimulation generate its own specific neural responses? Neuroimaging studies on acupuncture indicate the possible existence of neural correlates of acupuncture. ${ }^{95}$ Receptive fields of sensory neurons, important to determine perception sensitivity to a stimulus, are affected by receptor density at the stimulation area. We have recently found that $\alpha_{6} / \beta_{1}$ integrin receptors are highly expressed at the zusanli acupoint after acupuncture stimulation. ${ }^{35}$ Since integrin activity plays an important role in mediating intercellular signaling, ${ }^{96}$ it will be interesting to explore the distribution and activation of specific types of integrin receptors responding after stimulation at other acupoints. Moreover, it will be of great importance to investigate whether EA manipulation at the acupoint can generate a unique sensory modality, eg, deqi is interpreted as a special sensation distinct from typical somatosensation, which is shared between acupuncturist and patient. EAS induces afferent signals, and may directly affect visceral autonomic nerve activity. Alternatively, it is transmitted to the cerebrum and generates brain acupuncture signals (Figure 2). We speculate that acupuncture stimulation may trigger the responsiveness of sensory receptors and generate neural activity in its own specific way, which may be encoded in the cerebral cortex and autonomic neuronal center and exert its effects on regulating inflammation. Future investigations to explore whether acupuncture-specific vagal activity exists and acts on cells in target organs will be of great importance to gain insights into the mechanistic basis of acupuncture.

\section{Acknowledgment}

This research was supported by a grant of the Korea Health Technology R\&D Project through the Korea Health Industry Development Institute (KHIDI), funded by the Ministry of Health \& Welfare, Republic of Korea (grant number: HI17C0096).

\section{Disclosure}

The authors report no conflicts of interests in this work.

\section{References}

1. Zhu W, Ye Y, Liu Y et al. Mechanisms of acupuncture therapy for cerebral ischemia: an evidence-based review of clinical and animal studies on cerebral ischemia. $J$ Neuroimmune Pharmacol. 2017;12(4):575-592.

2. Shi P, Sun LL, Lee YS, Tu Y. Electroacupuncture regulates the stressinjury-repair chain of events after cerebral ischemia/reperfusion injury. Neural Regen Res. 2017;12(6):925-930.

3. Park JY, Kim YK, Kim SY, et al. Acupuncture modulates brain neural activity in patients: a systematic review and meta-analysis. Orient Pharm Exp Med. 2017;17(2):111-126.

4. World Health Organization. WHO International Standard Terminologies on Traditional Medicine in the Western Pacific Region. Geneva: WHO; 2007.

5. Stux G, Pomeranz B. Acupuncture: Textbook and Atlas. Heidelberg: Springer; 2012.

6. Ma W, Li Z, Lu Z, et al. Protective effects of acupuncture in cardiopulmonary bypass-induced lung injury in rats. Inflammation. 2017;40(4): 1275-1284.

7. Kong J, Gollub R, Huang T, et al. Acupuncture de qi, from qualitative history to quantitative measurement. J Altern Complement Med. 2007;13(10):1059-1070.

8. Kim DH. Huangdi's Internal Classic. Seoul: Euiseongdang; 2002.

9. White P, Bishop F, Hardy H, et al. Southampton needle sensation questionnaire: development and validation of a measure to gauge acupuncture needle sensation. JAltern Complement Med. 2008;14(4):373-379.

10. Park H, Park J, Lee H, Lee H. Does deqi (needle sensation) exist? Am $J$ Chin Med. 2002;30(1):45-50.

11. Kong J, Fufa DT, Gerber AJ, et al. Psychophysical outcomes from a randomized pilot study of manual, electro, and sham acupuncture treatment on experimentally induced thermal pain. J Pain. 2005;6(1): 55-64.

12. MacPherson H, Asghar A. Acupuncture needle sensations associated with de qi: a classification based on experts' ratings. JAltern Complement Med. 2006;12(7):633-637.

13. Jin $\mathrm{H}$, Guo J, Liu J, et al. Anti-inflammatory effects and mechanisms of vagal nerve stimulation combined with electroacupuncture in a rodent model of TNBS-induced colitis. Am J Physiol Gastrointest Liver Physiol. 2017;313(3):G192-G202.

14. Zhang R, Lao L, Ren K, Berman BM. Mechanisms of acupuncture-electroacupuncture on persistent pain. Anesthesiology. 2013;120(2):482-503.

15. Lin JG, Chen WL. Acupuncture analgesia: a review of its mechanisms of actions. Am J Chin Med. 2008;36(4):635-645. 
16. Takeuchi O, Akira S. Pattern recognition receptors and inflammation. Cell. 2010;140(6):805-820.

17. Borovikova LV, Ivanova $\mathrm{S}$, Zhang $\mathrm{M}$, et al. Vagus nerve stimulation attenuates the systemic inflammatory response to endotoxin. Nature. 2000;405(6785):458-462.

18. Wang H, Yu M, Ochani M, et al. Nicotinic acetylcholine receptor $\alpha 7$ subunit is an essential regulator of inflammation. Nature. 2003;421(6921):384-388.

19. de Jonge WJ, van der Zanden EP, Bijlsma MF, et al. Stimulation of the vagus nerve attenuates macrophage activation by activating the Jak2-STAT3 signaling pathway. Nat Immunol. 2005;6(8):844-851.

20. Wang H, Liao H, Ochani M, et al. Cholinergic agonists inhibit HMGB1 release and improve survival in experimental sepsis. Nat Med. 2004;10(11):1216-1221.

21. Tracey KJ. The inflammatory reflex. Nature. 2002;420(6917):853-859.

22. Rosas-Ballina M, Ochani M, Parrish WR, et al. Splenic nerve is required for cholinergic antiinflammatory pathway control of TNF in endotoxemia. Proc Natl Acad Sci U S A. 2008;105(31):11008-11013.

23. Rosas-Ballina M, Olofsson PS, Ochani M, et al. Acetylcholine-synthesizing T cells relay neural signals in a vagus nerve circuit. Science. 2011;334(6052):98-101.

24. Chiu IM, von Hehn CA, Woolf CJ. Neurogenic inflammation and the peripheral nervous system in host defense and immunopathology. Nat Neurosci. 2012;15(8):1063-1067.

25. Ghia JE, Blennerhassett P, Collins SM. Impaired parasympathetic function increases susceptibility to inflammatory bowel disease in a mouse model of depression. J Clin Invest. 2008;118(6):2209-2218.

26. Bockx I, Verdrengh K, Vander Elst I, et al. High-frequency vagus nerve stimulation improves portal hypertension in cirrhotic rats. Gut. 2012;61(4):604-612.

27. Pereira MR, Leite PE. The Involvement of parasympathetic and sympathetic nerve in the inflammatory reflex. J Cell Physiol. 2016;231(9): $1862-1869$

28. Koo A, Liang I. Microvascular filling pattern in rat liver sinusoids during vagal stimulation. J Physiol. 1979;295(1):191-199.

29. Koo A, Liang I. Parasympathetic cholinergic vasodilator mechanism in the terminal liver microcirculation in rats. Exp Physiol. 1979;64(3): 149-159.

30. Bellono NW, Bayrer JR, Leitch DB, et al. Enterochromaffin cells are gut chemosensors that couple to sensory neural pathways. Cell. 2017;170(1):185-198.e16.

31. Martelli D, McKinley MJ, McAllen RM. The cholinergic anti-inflammatory pathway: a critical review. Auton Neurosci. 2014;182:65-69.

32. Martelli D, Yao ST, McKinley MJ, McAllen RM. Reflex control of inflammation by sympathetic nerves, not the vagus. $J$ Physiol. 2014;592(7):1677-1686.

33. Wong CH, Jenne CN, Lee WY, Leger C, Kubes P. Functional innervation of hepatic iNKT cells is immunosuppressive following stroke. Science. 2011;334(6052):101-105.

34. Langevin HM, Churchill DL, Cipolla MJ. Mechanical signaling through connective tissue: a mechanism for the therapeutic effect of acupuncture. FASEB J. 2001;15(12):2275-2282.

35. Shin HC, Park JY, Lim HD, Namgung U. Induction of $\alpha_{6}$ and $\beta_{1}$ integrins by acupuncture stimulation in rats. Biochem Biophys Res Commun. 2017;491(3):629-635.

36. Lux G, Hagel J, Bäcker P, et al. Acupuncture inhibits vagal gastric acid secretion stimulated by sham feeding in healthy subjects. Gut. 1994;35(8): 1026-1029.

37. Liu Z, Liu Y, Xu H, et al. Effect of electroacupuncture on urinary leakage among women with stress urinary incontinence: a randomized clinical trial. JAMA. 2017;317(24):2493-2501.

38. Wu XK, Stener-Victorin E, Kuang HY, et al. Effect of acupuncture and clomiphene in Chinese women with polycystic ovary syndrome: a randomized clinical trial. JAMA. 2017;317(24):2502-2514.

39. Lim HD, Kim MH, Lee CY, Namgung U. Anti-inflammatory effects of acupuncture stimulation via the vagus nerve. PloS One. 2016;11(3): $\mathrm{e} 0151882$.
40. da Silva MD, Bobinski F, Sato KL, Kolker SJ, Sluka KA, Santos AR. IL-10 cytokine released from M2 macrophages is crucial for analgesic and anti-inflammatory effects of acupuncture in a model of inflammatory muscle pain. Mol Neurobiol. 2015;51(1):19-31.

41. Son YS, Park HJ, Kwon OB, Jung SC, Shin HC, Lim S. Antipyretic effects of acupuncture on the lipopolysaccharide-induced fever and expression of interleukin- 6 and interleukin- $1 \beta$ mRNAs in the hypothalamus of rats. Neurosci Lett. 2002;319(1):45-48.

42. Ceccherelli F, Gagliardi G, Matterazzo G, Visentin R, Giron G. The role of manual acupuncture and morphine administration on the modulation of capsaicin-induced edema in rat paw: a blind controlled study. Acupunct Electrother Res. 1996;21(1):7-14.

43. Wang K, Zhang R, Xiang X, et al. Differences in neural-immune gene expression response in rat spinal dorsal horn correlates with variations in electroacupuncture analgesia. PLoS One. 2012;7(8):e42331.

44. Lu KW, Hsu CK, Hsieh CL, Yang J, Lin YW. Probing the effects and mechanisms of electroacupuncture at ipsilateral or contralateral ST36-ST37 acupoints on CFA-induced inflammatory pain. Sci Rep. 2016;6:22123.

45. Liao HY, Hsieh CL, Huang CP, Lin YW. Electroacupuncture attenuates CFA-induced inflammatory pain by suppressing Nav1.8 through S100B, TRPV1, opioid, and adenosine pathways in mice. Sci Rep. 2017;7:42531.

46. Li A, Lao L, Wang Y, et al. Electroacupuncture activates corticotrophinreleasing hormone-containing neurons in the paraventricular nucleus of the hypothalamus to alleviate edema in a rat model of inflammation. BMC Complement Altern Med. 2008;8:20.

47. Baek YH, Choi DY, Yang HI, Park DS. Analgesic effect of electroacupuncture on inflammatory pain in the rat model of collagen-induced arthritis: mediation by cholinergic and serotonergic receptors. Brain Res. 2005;1057(1-2):181-185.

48. Li J, Li J, Chen R, Cai G. Targeting NF- $\kappa$ and TNF- $\alpha$ activation by electroacupuncture to suppress collagen-induced rheumatoid arthritis in model rats. Altern Ther Health Med. 2015;21(4):26-34.

49. Tian L, Huang YX, Tian M, Gao W, Chang Q. Downregulation of electroacupuncture at ST36 on TNF- $\alpha$ in rats with ulcerative colitis. World J Gastroenterol. 2003;9(5):1028-1033.

50. Gondim DV, Costa JL, Rocha SS, Brito GA, Ribeiro RA, Vale ML. Antinociceptive and anti-inflammatory effects of electroacupuncture on experimental arthritis of the rat temporomandibular joint. Can J Physiol Pharmacol. 2012;90(4):395-405.

51. Liu F, Fang J, Shao X, Liang Y, Wu Y, Jin Y. Electroacupuncture exerts an anti-inflammatory effect in a rat tissue chamber model of inflammation via suppression of NF- $\mathrm{KB}$ activation. Acupunct Med. 2014;32(4):340-345.

52. Zhang D, Song XJ, Li SY, et al. Evaluation of liver function and electroacupuncture efficacy of animals with alcoholic liver injury by the novel imaging methods. Sci Rep. 2016;6:30119.

53. Song JG, Li HH, Cao YF, et al. Electroacupuncture improves survival in rats with lethal endotoxemia via the autonomic nervous system. Anesthesiology. 2012;116(2):406-414.

54. Goldman N, Chen M, Fujita T, et al. Adenosine A1 receptors mediate local anti-nociceptive effects of acupuncture. Nat Neurosci. 2010;13(7):883-888.

55. Tu WZ, Cheng RD, Cheng B, et al. Analgesic effect of electroacupuncture on chronic neuropathic pain mediated by $\mathrm{P} 2 \mathrm{X} 3$ receptors in rat dorsal root ganglion neurons. Neurochem Int. 2012;60(4): 379-386.

56. Ji RR, Zhang ZW, Zhou Y, Zhang Q, Han JS. Induction of c-Fos expression in the rostral medulla of rats following electroacupuncture stimulation. Int J Neurosci. 1993;72(3-4):183-191.

57. Liu JH, Li J, Yan J, et al. Expression of c-Fos in the nucleus of the solitary tract following electroacupuncture at facial acupoints and gastric distension in rats. Neurosci Lett. 2004;366(2):215-219.

58. Kim SK, Kim J, Woo HS, et al. Electroacupuncture induces Fos expression in the nucleus tractus solitarius via cholecystokinin A receptor signaling in rats. Neurol Res. 2010;32 Suppl 1:116-119. 
59. Takahashi T. Acupuncture for functional gastrointestinal disorders. $J$ Gastroenterol. 2006;41(5):408-417.

60. Yin J, Chen JD. Gastrointestinal motility disorders and acupuncture. Auton Neurosci. 2010;157(1):31-37.

61. Ouyang H, Yin J, Wang Z, Pasricha PJ, Chen JD. Electroacupuncture accelerates gastric emptying in association with changes in vagal activity. Am J Physiol Gastrointest Liver Physiol. 2002;282(2):G390-G396.

62. Sakai S, Hori E, Umeno K, Kitabayashi N, Ono T, Nishijo H. Specific acupuncture sensation correlates with EEGs and autonomic changes in human subjects. Auton Neurosci. 2007;133(2):158-169.

63. Longhurst JC, Tjen-A-Looi S. Acupuncture regulation of blood pressure: two decades of research. Int Rev Neurobiol. 2013;111:257-271.

64. Zhang WT, Jin Z, Cui GH, et al. Relations between brain network activation and analgesic effect induced by low vs. high frequency electrical acupoint stimulation in different subjects: a functional magnetic resonance imaging study. Brain Res. 2003;982(2): $168-178$.

65. Napadow V, Makris N, Liu J, Kettner NW, Kwong KK, Hui KK. Effects of electroacupuncture versus manual acupuncture on the human brain as measured by fMRI. Hum Brain Mapp. 2005;24(3):193-205.

66. Fang JF, Fang JQ, Shao XM, et al. Electroacupuncture treatment partly promotes the recovery time of postoperative ileus by activating the vagus nerve but not regulating local inflammation. Sci Rep. 2017; 7:39801.

67. Hu Z, Chen B, Tong J, Huang D, Lu S. The change of C-Fos expression in ovariectomized rats following electroacupuncture treatment: an immunohistochemistry study. Acupunct Electrother Res. 1993;18(2):117-124.

68. Torres-Rosas R, Yehia G, Peña G, et al. Dopamine mediates vagal modulation of the immune system by electroacupuncture. Nat Med. 2014;20(3):291-295.

69. Zhang J, Yong Y, Li X, et al. Vagal modulation of high mobility group box-1 protein mediates electroacupuncture-induced cardioprotection in ischemia-reperfusion injury. Sci Rep. 2015;5:15503.

70. Chen J, Song GQ, Yin J, Koothan T, Chen JD. Electroacupuncture improves impaired gastric motility and slow waves induced by rectal distension in dogs. Am J Physiol Gastrointest Liver Physiol. 2008;295(3):G614-G620.

71. Yu Z, Zhang N, Lu CX, et al. Electroacupuncture at ST25 inhibits jejunal motility: role of sympathetic pathways and TRPV1. World $J$ Gastroenterol. 2016;22(5):1834-1843.

72. Benrick A, Kokosar M, Hu M, et al. Autonomic nervous system activation mediates the increase in whole-body glucose uptake in response to electroacupuncture. FASEB J. 2017;31(8):3288-3297.

73. Tjen-A-Looi SC, Guo ZL, Fu LW, Longhurst JC. Paraventricular nucleus modulates excitatory cardiovascular reflexes during electroacupuncture. Sci Rep. 2016;6:25910.

74. Kim HW, Kang SY, Yoon SY, et al. Low-frequency electroacupuncture suppresses zymosan-induced peripheral inflammation via activation of sympathetic post-ganglionic neurons. Brain Res. 2007;1148:69-75.

75. Kim HW, Uh DK, Yoon SY, et al. Low-frequency electroacupuncture suppresses carrageenan-induced paw inflammation in mice via sympathetic post-ganglionic neurons, while high-frequency EA suppression is mediated by the sympathoadrenal medullary axis. Brain Res Bull. 2008;75(5):698-705.

76. Chavez LM, Huang SS, MacDonald I, Lin JG, Lee YC, Chen YH. Mechanisms of acupuncture therapy in ischemic stroke rehabilitation: a literature review of basic studies. Int J Mol Sci. 2017;18(11):E2270.

77. Liu F, Jiang YJ, Zhao HJ, Yao LQ, Chen LD. Electroacupuncture ameliorates cognitive impairment and regulates the expression of apoptosis-related genes Bcl-2 and Bax in rats with cerebral ischaemiareperfusion injury. Acupunct Med. 2015;33(6):478-484.

78. Liu W, Wang X, Yang S, et al. Electroacupuncture improves motor impairment via inhibition of microglia-mediated neuroinflammation in the sensorimotor cortex after ischemic stroke. Life Sci. 2016; $151: 313-322$.
79. Yuan S, Zhang X, Bo Y, Li W, Zhang H, Jiang Q. The effects of electroacupuncture treatment on the postoperative cognitive function in aged rats with acute myocardial ischemia-reperfusion. Brain Res. 2014;1593:19-29.

80. Tao J, Zheng Y, Liu W, et al. Electro-acupuncture at LI11 and ST36 acupoints exerts neuroprotective effects via reactive astrocyte proliferation after ischemia and reperfusion injury in rats. Brain Res Bull. 2016;120:14-24.

81. Chen Y, Zhou J, Li J, et al. Electroacupuncture pretreatment prevents cognitive impairment induced by limb ischemia-reperfusion via inhibition of microglial activation and attenuation of oxidative stress in rats. Brain Res. 2012;1432:36-45.

82. Chuang CH, Hsu YC, Wang CC, Hu C, Kuo JR. Cerebral blood flow and apoptosis-associated factor with electroacupuncture in a traumatic brain injury rat model. Acupunct Med. 2013;31(4):395-403.

83. Zhu XL, Chen X, Wang W, et al. Electroacupuncture pretreatment attenuates spinal cord ischemia-reperfusion injury via inhibition of high-mobility group box 1 production in a LXA4 receptor-dependent manner. Brain Res. 2017;1659:113-120.

84. Wang Q, Wang F, Li X, et al. Electroacupuncture pretreatment attenuates cerebral ischemic injury through $\alpha 7$ nicotinic acetylcholine receptor-mediated inhibition of high-mobility group box 1 release in rats. J Neuroinflammation. 2012;9:24.

85. Schmidt AM, Yan SD, Yan SF, Stern DM. The multiligand receptor RAGE as a progression factor amplifying immune and inflammatory responses. J Clin Invest. 2001;108(7):949-955.

86. Xu H, Zhang Y, Sun H, Chen S, Wang F. Effects of acupuncture at GV20 and ST36 on the expression of matrix metalloproteinase 2, aquaporin 4, and aquaporin 9 in rats subjected to cerebral ischemia/ reperfusion injury. PLoS One. 2014;9(5):e97488.

87. Lv E, Deng J, Yu Y, et al. Nrf2-ARE signals mediated the anti-oxidative action of electroacupuncture in an MPTP mouse model of Parkinson's disease. Free Radic Res. 2015;49(11):1296-1307.

88. Zhou H, Zhang Z, Wei H, et al. Activation of STAT3 is involved in neuroprotection by electroacupuncture pretreatment via cannabinoid CB1 receptors in rats. Brain Res. 2013;1529:154-164.

89. Sun N, Zou X, Shi J, Liu X, Li L, Zhao L. Electroacupuncture regulates NMDA receptor NR1 subunit expression via PI3-K pathway in a rat model of cerebral ischemia-reperfusion. Brain Res. 2005;1064(1-2): 98-107.

90. Liu Z, Chen X, Gao Y, et al. Involvement of GluR2 up-regulation in neuroprotection by electroacupuncture pretreatment via cannabinoid CB1 receptor in mice. Sci Rep. 2015;5:9490.

91. Lu Y, Zhao H, Wang Y, et al. Electro-acupuncture up-regulates astrocytic MCT1 expression to improve neurological deficit in middle cerebral artery occlusion rats. Life Sci. 2015;134:68-72.

92. Chi L, Du K, Liu D, Bo Y, Li W. Electroacupuncture brain protection during ischemic stroke: a role for the parasympathetic nervous system. J Cereb Blood Flow Metab. 2018;38(3):479-491.

93. Gardner EP, Johnson KO. Principles of Neural Science. 5th ed. New York: McGraw-Hill; 2013.

94. Johnson KO. Neural coding. Neuron. 2000;26(3):563-566.

95. Dhond RP, Kettner N, Napadow V. Do the neural correlates of acupuncture and placebo effects differ? Pain. 2007;128(1-2):8-12.

96. Hynes RO. Integrins: bidirectional, allosteric signaling machines. Cell. 2002;110(6):673-687.

97. Geng Y, Chen D, Zhou J, et al. Synergistic effects of electroacupuncture and mesenchymal stem cells on intestinal ischemia/reperfusion injury in rats. Inflammation. 2016;39(4):1414-1420.

98. Fang B, Qin M, Li Y, et al. Electroacupuncture preconditioning and postconditioning inhibit apoptosis and neuroinflammation induced by spinal cord ischemia reperfusion injury through enhancing autophagy in rats. Neurosci Lett. 2017;642:136-141.

99. Ho TY, Lo HY, Chao DC, et al. Electroacupuncture improves trinitrobenzene sulfonic acid-induced colitis, evaluated by transcriptomic study. Evid Based Complement Alternat Med. 2014;2014:942196. 
100. Song XM, Wu XJ, Li JG, et al. The effect of electroacupuncture at ST36 on severe thermal injury-induced remote acute lung injury in rats. Burns. 2015;41(7):1449-1458.

101. Wang K, Ju Z, Yong Y, Chen T, Song J, Zhou J. The effects of electroacupuncture on the apelin/APJ system in the spinal cord of rats with inflammatory pain. Anesth Analg. 2016;123(6):1603-1610.

102. Dong ZQ, Zhu J, Lu DZ, Chen Q, Xu YL. Effect of electroacupuncture in "zusanli" and "kunlun" acupoints on TLR4 signaling pathway of adjuvant arthritis rats. Am J Ther. Epub 2016 Aug 29.

103. Zhang H, He S, Hu Y, Zheng H. Antagonism of cannabinoid receptor 1 attenuates the anti-inflammatory effects of electroacupuncture in a rodent model of migraine. Acupunct Med. 2016;34(6):463-470.

104. Chen Y, Lei Y, Mo LQ, et al. Electroacupuncture pretreatment with different waveforms prevents brain injury in rats subjected to cecal ligation and puncture via inhibiting microglial activation, and attenuating inflammation, oxidative stress and apoptosis. Brain Res Bull. 2016;127:248-259.

105. Liaw JJ, Peplow PV. Effect of electroacupuncture on inflammation in the obese Zucker fatty rat model of metabolic syndrome. J Acupunct Meridian Stud. 2016;9(2):73-79.

106. Wen CK, Lee TY. Electroacupuncture prevents white adipose tissue inflammation through modulation of hypoxia-inducible factors- $1 \alpha$ dependent pathway in obese mice. BMC Complement Altern Med. $2015 ; 15: 452$.
107. Zhu J, Chen XY, Li LB, et al. Electroacupuncture attenuates collageninduced arthritis in rats through vasoactive intestinal peptide signalling-dependent re-establishment of the regulatory T cell/T-helper 17 cell balance. Acupunct Med. 2015;33(4):305-311.

108. Lisboa MR, Gondim DV, Ervolino E, et al. Effects of electroacupuncture on experimental periodontitis in rats. $J$ Periodontol. 2015;86(6):801-811.

109. Han D, Liu Z, Wang G, Zhang Y, Wu Z. Electroacupuncture improves cognitive deficits through increasing regional cerebral blood flow and alleviating inflammation in CCI rats. Evid Based Complement Alternat Med. 2017;2017:5173168.

110. Shen MH, Zhang CB, Zhang JH, Li PF. Electroacupuncture attenuates cerebral ischemia and reperfusion injury in middle cerebral artery occlusion of rat via modulation of apoptosis, inflammation, oxidative stress, and excitotoxicity. Evid Based Complement Alternat Med. 2016;2016:9438650.

111. Liu W, Wang X, Zheng Y, et al. Electroacupuncture inhibits inflammatory injury by targeting the miR-9-mediated NF- $\kappa \mathrm{B}$ signaling pathway following ischemic stroke. Mol Med Rep. 2016;13(2): 1618-1626.

112. Lan L, Tao J, Chen A, et al. Electroacupuncture exerts anti-inflammatory effects in cerebral ischemia-reperfusion injured rats via suppression of the TLR4/NF-KB pathway. Int J Mol Med. 2013;31(1) 75-80.
Journal of Inflammation Research

\section{Publish your work in this journal}

The Journal of Inflammation Research is an international, peer-reviewed open access journal that welcomes laboratory and clinical findings on the molecular basis, cell biology and pharmacology of inflammation including original research, reviews, symposium reports, hypothesis formation and commentaries on: acute/chronic inflammation; mediators of

\section{Dovepress}

inflammation; cellular processes; molecular mechanisms; pharmacology and novel anti-inflammatory drugs; clinical conditions involving inflammation. The manuscript management system is completely online and includes a very quick and fair peer-review system. Visit http://www.dove press.com/testimonials.php to read real quotes from published authors. 